\title{
Remarques sur le fragment L 147 : Pascal et Jansénius contre les stoïciens
}

Chiara Catalano

\section{OpenEdition}

\section{Journals}

Édition électronique

URL : http://journals.openedition.org/ccibp/275

DOI : $10.4000 /$ ccibp. 275

ISSN : 2493-7460

Éditeur

Centre international Blaise Pascal

\section{Édition imprimée}

Date de publication : 1 décembre 2012

Pagination : 7-12

ISBN : 978-2-84516-612-7

ISSN : 0249-6674

Référence électronique

Chiara Catalano, «Remarques sur le fragment L 147 : Pascal et Jansénius contre les stoïciens », Courrier du Centre international Blaise Pascal [En ligne], 34 | 2012, mis en ligne le 03 décembre 2015, consulté le 01 mai 2019. URL : http://journals.openedition.org/ccibp/275; DOI : 10.4000/ccibp.275

Ce document a été généré automatiquement le 1 mai 2019

Centre international Blaise Pascal 


\title{
Remarques sur le fragment L 147 : Pascal et Jansénius contre les stoïciens
}

\author{
Chiara Catalano
}

\section{NOTE DE L'AUTEUR}

J'utilise dans cette étude les abréviations suivantes:

[HP] Cornelis JANSEN, De hoeresi pelagiana, dans Augustinus, seu Doctrina Sancti Augustini de humance naturce sanitate, cegritudine, medicina adversus Pelagianos et Massilienses, tribus tomis comprehensa, Lovanii, typis et sumptibus Iacobi Zegeri, 1640, I, 1-557

[Lov.] Opera D. Aurelii Augustini Hipponensis episcopi et doctoris precipui, tomis decem comprehensa: per theologos lovanienses ex manuscriptis codicibus multo labore emendata, et ab innumeris erroribus vindicata: illustrata preterea eruditis censuris, et locupletata multis homiliis et aliquot epistolis eiusdem B. Augustini, antea non editis: cum indice tripartito, Antverpiæ, Apud Christophorum Plantinum, 1576-1577.

[SPN] Cornelis JANSEN, De statu purce naturce, dans Augustinus, II, 677-982.

1 Dans la première partie de la dixième liasse des Pensées, intitulée Le Souverain Bien, c'est-àdire dans le fragment Dispute du souverain bien (L 147 / B 361) ${ }^{1}$, Pascal écrit :

Le Souverain Bien. Dispute du souverain bien.

Ut sis contentus temetipso et ex te nascentibus bonis.

Il y a contradiction, car ils conseillent enfin de se tuer.

Oh ! quelle vie heureuse dont on se délivre comme de la peste!

2 En critiquant les philosophes stoïques, la liasse suit le fil de la précédente, la neuvième, intitulée Philosophes ${ }^{2}$ et composée de sept fragments. Elle est également liée au fragment Stoïques ${ }^{3}$, où la véritable cible polémique est déclarée ${ }^{4}$. En effet, dans le fragment L 147 Pascal fait encore référence aux stoïciens ; et si le fragment précédent (L 146) se termine sur une référence à Épictète [Entretiens, IV, 7], il s'ouvre par une crypto-citation de Sénèque, tirée du huitième chapitre de la vingtième Epistola ad Lucilium. Ensuite, Pascal 
remarque la contradiction des stoïciens, qui conseillent de se tuer tout en considérant la vie comme heureuse, et termine le fragment par une interjection qui renvoie à un passage de l'Epistola 155 d'Augustin.

Cette même épître est citée par Jansénius dans le huitième chapitre du second livre $D e$ statu purce naturce de l'Augustinus. En outre, dans le premier chapitre du cinquième livre De horesi pelagiana figure aussi le même passage de Sénèque, qui paraît dans le fragment de Pascal.

Je me propose ici d'établir une comparaison entre le fragment de la liasse Le Souverain Bien et les passages de Jansénius afin de démontrer la présence d'éléments spécifiquement de Jansénius (et pas seulement d'Augustin) à l'intérieur du texte de Pascal. Il me semble que la proximité des textes de Jansénius et de Pascal peut être montrée par quelques éléments objectifs, tels que la ressemblance du contexte polémique antistoïcien - surtout en ce qui concerne le chapitre du cinquième livre De horesi pelagiana - et la source commune augustinienne, relevant du huitième chapitre du second livre De statu purce naturce. Mon argumentation se développera en trois parties :

1. Analyse de la citation de Sénèque (Epistola ad Lucilium 20) afin d'observer les similitudes et les différences des deux contextes de discussion et, en particulier, des solutions trouvées par Jansénius et Pascal ;

2. Comparaison entre Jansénius et Pascal par rapport à un passage extrait de l'Epistola 155 d'Augustin ;

3. Analyse du thème stoïcien du suicide dans Jansénius et Pascal et comparaison avec la source augustinienne commune.

Il faut dire tout d'abord que le rapport entre le fragment de Pascal et l'Augustinus a déjà fait l'objet d'analyses de certains commentateurs. Celle qui, à mon avis, résume le mieux cet aspect est proposée par Philippe Sellier qui, dans son livre Pascal et Saint Augustin de $1970^{5}$, en remarquant que la ressemblance entre le texte de Jansénius et celui de Pascal est due principalement à leur source commune, c'est-à-dire à l'Epistola 155 d'Augustin, tire des conclusions qui peuvent être schématiquement résumées comme suit :

1. Le titre de la liasse (Le Souverain Bien) et celui du fragment (L 147; Dispute du Souverain Bien) renvoient au sujet traité par Jansénius dans les neuf premiers chapitres du second livre De statu purce naturce du second tome de l'Augustinus ${ }^{6}$.

2. Pascal utilise le même passage de Sénèque qui est contenu dans le premier chapitre du cinquième livre De horesi pelagiana ${ }^{7}$.

3. Pascal « se souvient ${ }^{8} »$, pour composer son fragment, d'un passage tiré du huitième chapitre du second livre De statu purce nature.

4. Pascal et Jansénius utilisent tous deux l'Epistola 155 d'Augustin.

Or, il faut remarquer que dans le premier chapitre du cinquième livre du De hoeresi pelagiana, Jansénius caractérise le premier des quatre états du pélagianisme ${ }^{9}$, c'est-à-dire l'ethnicisme, comme celui qui exalte la nature nue ${ }^{10}$. Jansénius considère l'ethnicisme comme un simple rêve de la philosophie ethnique, plutôt une sorte de paganisme qu'une hérésie ${ }^{11}$, et il polémique contre les philosophes, surtout stoïciens, qui ont soutenu que l'homme peut acquérir tout seul, sans l'aide de Dieu, la vertu, la sagesse et le bonheur :

«Quid enim egerunt tanto disputationum impetu Philosophi huius mundi maximeque Stoici nisi ut hominem ipsum sibi repudiato omni sive Dei sive alterius adiutorio, ad omnem virtutem, sapientiam, felicitatemque sufficere persuaderent ${ }^{12}$ ?». 
7 Jansénius reconnaît dans l'idée stoïcienne d'autosuffisance de l'homme - qui peut tout seul atteindre à la vertu, à la sagesse et au bonheur - une manifestation du paganisme, qui est le premier état de l'hérésie de Pélage. Il suggère que cette idée a été reprise par Pélage et qu'elle est présente en Sénèque. Pour le démontrer, il cite d'abord l'Epistola $53^{13}$, puis l'Epistola 20.

8 Il faut remarquer ici, avant tout, que la seconde citation est la même que celle utilisée par Pascal dans le fragment L 147. En particulier, en lisant le passage de Jansénius - qui est situé environ à la fin du premier chapitre du cinquième livre De horesi pelagiana, à la fin de la seconde colonne -, on peut constater que l'auteur, au contraire de Pascal, renvoie explicitement à Sénèque :

«Ecce suo beneficio non Dei hominem sapientem fieri putat. Et alibi : Huc cogitationes tuæ tendant, hoc cura, hoc opta omnia alia vota Deo remissurus, ut contentus sis temetipso et ex te nascentibus bonis. Quæ potest esse felicitas Deo propior? [SENECA, Epistola ad Lucilium XX, 8] Quasi cæterorum bonorum vota Deo essent remittenda, ad parandas vero animi opes solus homo sibi ipse sufficeret ${ }^{14} »$.

9 Comme on peut voir, la citation de l'épître de Sénèque est ici plus longue et plus complète; elle est aussi restituée dans un contexte plus large que dans le fragment L 147. En effet, le théologien de Louvain, après avoir commenté l'Epistola 53 de Sénèque et en notant que la sagesse humaine n'est pas due à Dieu, passe à l'analyse de l'Epistola 20 et insiste sur l'idée de l'autonomie de l'homme dans le souci du bonheur personnel et des biens relatifs. Il résume ensuite la thèse de Sénèque: selon le stoïcien les occupations de l'âme appartiennent à l'homme seulement, alors que tous les autres biens ne dépendent que de Dieu.

10 Comme on sait, Jansénius reproche aux pélagiens à plusieurs reprises (pas seulement dans ce chapitre ${ }^{15}$ ) d'avoir soutenu la suffisance de la nature humaine pure, dépourvue de toute aide, tant en termes de connaissance que de pouvoir. Ils reconnaissent la seule contribution du libre arbitre, de la volonté et de la possibilitas naturelle dans tout ce qui est important pour une vie bonne et heureuse. En effet, peu après le début du chapitre, au milieu de la première colonne, il écrit :

«Tunc enim puram putamque naturam sicut a Deo conditur, et ex utero matris prodit, nullo externo sive scientiæ sive potentiæ munitam præsidio, sed solo liberæ voluntatis arbitrio et naturali illa possibilitate, ad omnia omnino quæ ad bonam beatamque vitam magna facilitate consequendam pertinent, abunde sufficere docuerunt ${ }^{16} \%$.

11 Or, pour revenir à l'interprétation proposée par Philippe Sellier ${ }^{17}$, qui reconnaît dans le titre du fragment et de la liasse Le Souverain Bien un renvoi à la thématique traitée par Jansénius dans les chapitres 1-9 du second livre du De statu purce naturee, il faut faire ici deux remarques.

12 Premièrement, cette thématique fait l'objet d'un traitement par Jansénius pas seulement dans ces chapitres, mais aussi dans de nombreux autres du second tome de l'Augustinus, que je ne peux pas ici citer en entier ${ }^{18}$. Je voudrais brièvement indiquer, par exemple, les dixième, douzième et treizième chapitres du quatrième livre De statu naturce lapsce; le quinzième du premier livre, le cinquième du second livre et le quinzième du troisième livre De statu purce naturce, en invitant le lecteur à les lire par lui-même.

Deuxièmement, bien que ce sujet caractérise toute la dixième liasse - et aussi la seconde partie (L 148), où on retrouve le lien entre le souverain bien et le bonheur ${ }^{19}-\mathrm{il}$ semble qu'il n'y ait aucune référence explicite au chapitre de Jansénius, à l'exception de l'usage 
commun de la source augustinienne dans le fragment L 147, comme le fait observer Sellier lui-même: Jansénius dans le huitième chapitre, tout comme Pascal dans le fragment L 147, utilise la lettre 155 (52) d'Augustin.

C'est en particulier cette interjection de Pascal (L 147) qui renvoie à la lettre en question :

« Oh ! quelle vie heureuse dont on se délivre comme de la peste $! »^{20}$.

Dans le tableau qui suit, je signale les différences et les analogies entre Jansénius et Pascal par rapport au passage augustinien :

\begin{tabular}{|c|c|c|}
\hline AUGUSTINUS, Epistola 155 (52) & $\begin{array}{l}\text { JANSENIUS, Augustinus, De statu purce } \\
\text { naturce, II, VIII, col. 821A-B }\end{array}$ & $\begin{array}{l}\text { PASCAL, Pensées, liasse X, L } \\
147\end{array}$ \\
\hline $\begin{array}{l}\text { "vel ideo beatus, quoniam } \\
\text { fuerit ab illa beata vita, tanquam } \\
a b \text { aliqua peste liberatus }{ }^{21} \text {. }\end{array}$ & $\begin{array}{l}\text { «O vitam scilicet beatissimam } \\
\text { sapientis, qua ut fruatur mortis } \\
\text { quærit auxilium!» }\end{array}$ & $\begin{array}{llll}\text { «Oh! quelle } & & \text { vie } \\
\text { heureuse dont on } & \text { se } \\
\text { délivre comme de la } & \text { la } \\
\text { peste!» } & & & \end{array}$ \\
\hline
\end{tabular}

16 Nous pouvons constater une plus grande fidélité du texte de Pascal à celui d'Augustin, en même temps qu'une ressemblance entre Pascal et Jansénius : c'est d'ailleurs normal, si l'on considère que tant Pascal que Jansénius renvoient à la source augustinienne. Ce qui différencie les deux textes est l'absence chez Jansénius de la métaphore augustinienne de la vie considérée comme peste, qui est en revanche contenue dans celui de Pascal. D'ailleurs, si l'on compare les deux textes de Pascal et de Jansénius sur le plan stylistique, la ressemblance est frappante : ils utilisent, l'un et l'autre, des interjections.

17 On peut considérer cette preuve comme faible et insuffisante pour établir que Pascal a effectivement utilisé le passage de Jansénius. Cependant, je pense qu'on peut répondre par l'affirmative à cette question. Pour le faire, il faut commencer par analyser le contexte du passage de Jansénius à partir du début du chapitre environ jusqu'à l'interjection en question.

18 Le huitième chapitre du second livre du De statu purce naturce s'intitule Ostenditur insania Philosophorum naturalem beatitudinem sibi machinantium in ea quam putabant esse puram naturam. Superbia singularis tantum naturce etiam purce viribus tribuere. Jansénius poursuit ici sa critique de l'état de la nature pure. En effet, dans le premier chapitre du livre, il a développé sa deuxième argumentation contre cet état (à partir de la fruitio beatifica du souverain bien ${ }^{22}$, après avoir présenté la première ${ }^{23}$ dans le troisième chapitre du premier livre).

19 Il s'agit bien sûr d'un contexte augustinien dans lequel Jansénius traite de l'ordre des créatures rationnelles et de leur amour de Dieu, de leurs principes et de leur fin. Il commence donc ce premier chapitre par l'idée de fruitio Dei tanquam beatificum bonum : l'homme, en tant que créature rationnelle, ne peut pas être juste et bon par lui-même et sans l'amour de Dieu. Il écrit :

«Secundum igitur argumentum principale contra statum puræ naturæ peti potest ex parte fruitionis Dei, tanquam beatifici boni. Creatura enim rationalis, non solum recta et bona esse non potest, sine dilectione Dei, quæ dilectio voluntatis est quasi rectus sagittæ motus ad scopi percussionem, aptaque dispositio voluntatis velut materiæ ad susceptionem beatitudinis tanquam formæ suæ; sed neque beari sine contemplatione velut comprehensione eiusdem Dei potest. Est enim anima rationalis naturaliter, hoc ipso quo rationalis et imago Dei est, tam immensæ 
capacitatis, ut nulla re alia satietur, et per hoc neque beetur nisi sola immensitate $\operatorname{Dei}^{24} »$.

20 L'âme, qui est par nature rationnelle et qui est aussi l'image de Dieu, a une si grande capacité qu'elle ne peut être heureuse et rassasiée que par l'immensité de Dieu. En utilisant la doctrine augustinienne, Jansénius affirme que l'amour de la volonté (dilectio voluntatis) est une disposition de la volonté (dispositio voluntatis) à accueillir le bonheur ( susceptio beatitudinis), comme la matière à la forme. Jansénius se réfère ici à deux passages de saint Augustin: l'un tiré du septième chapitre du De natura boni ${ }^{25}$ et l'autre tiré du huitième chapitre du quatorzième livre de De Trinitate $\mathrm{Dei}^{26}$.

21 L'analyse de ce passage révèle un contexte évidemment augustinien qui représente à la fois une source et un point de départ pour la discussion du sujet traité. C'est dans ce contexte que Jansénius présente ses argumentations contre la nature pure, notamment la deuxième dans le Livre II, chapitre I du De statu naturce purce ${ }^{27}$, la troisième dans le chapitre III ${ }^{28}$, la quatrième dans le chapitre $\mathrm{V}^{29}$, la cinquième et la sixième dans le chapitre $\mathrm{X}^{30}$. Il développe aussi dans le onzième chapitre une autre argumentation ex parte concupiscientioe, qui se compose de huit autres argumentations contenues dans les chapitres compris entre le onzième et le vingt-et-unième.

Dans le chapitre qui fait l'objet de mon analyse (SNP II, VIII) ${ }^{31}$, Jansénius se penche sur la folie des philosophes. Ils ont soutenu que l'homme peut gagner le bonheur (beatitudo) naturel par la seule nature pure. Mais qui sont-ils, ces philosophes ? Les stoïciens bien entendu, c'est-à-dire ceux-là mêmes que critique Pascal dans la dixième liasse. Jansénius souligne l'orgueil avec lequel ils n'attribuent de pouvoir qu'aux forces de la nature pure : il naît de l'erreur des philosophes païens, qui ont accordé au sage la possibilité d'acquérir le bonheur par les choses naturelles.

Jansénius parle d'une imaginatio du bonheur philosophique, qui est devenue commune aussi aux chrétiens et qui est fondée sur une certaine contemplation naturelle de Dieu ${ }^{32}$. $C^{\prime}$ est cette imagination qui a trompé les fondateurs de la notion de nature pure (conditores naturce purce $)^{33}$ et qui a donc été la cause de leurs erreurs. Il poursuit en écrivant :

«Stoici quippe sunt quibus præcipue typhus ille superbiæ placuit, ut se in hac misera sua quam putabant pura natura beatissimos esse posse sentirent $»^{34}$.

Voici les stoïciens qui aiment surtout l'arrogance de l'orgueil et qui croient pouvoir être heureux dans l'état de la nature pure - et donc grâce à leurs forces naturelles. Et en s'opposant à ces philosophes, il ajoute encore :

«Nam sapientem suum dixerunt sola virtute contentum ${ }^{35}$, etiam in Phalaridis tauro esse beatissimum, usque adeo ut nec illa quæ dicuntur mala, mala esse contendant, qua de re Tullius in Paradoxis ac Tusculanis videri potest ${ }^{36} »$.

Dans ce passage, Jansénius paraphrase ${ }^{37}$ l'épître 155 d'Augustin. En effet, il y a la même référence au taureau de Phalaris ${ }^{38}$ qui est présente dans le texte augustinien. En outre, Jansénius souligne que le sage stoïcien est satisfait de la seule vertu et que, grâce à elle, il est très heureux. À ce propos, il renvoie à Cicéron, c'est-à-dire aux Paradoxa et aux Tusculance disputationes.

Il écrit ensuite :

«Sed quid nisi magnus quidam stupor arrogantiæ huiusmodi paradoxa peperit, quandoquidem si tanta fuerint illa quæ dicuntur ab aliis mala, et ab ipsis bona, ut ea sapiens, qualem mirabili vanitate describunt, vel non possit vel non debeat sustinere, cogi eum sibi mortem inferre, et ex hac vita emigrare fateantur ? $»^{39}$. 
27 Jansénius soutient que les paradoxes de l'orgueil sont nés par un engourdissement qui a forcé le sage à se pendre. Bien que ce dernier considère les maux de la vie comme biens, comme le théologien de Louvain l'explique, c'est à cause de sa misérable vie qu'il décide de se tuer.

Plus loin, Jansénius cite un passage de Cicéron tiré des Paradoxa (II, 17) ${ }^{40}$, dans lequel l'orateur parle du bonheur de l'homme imbu de soi-même :

«Cedit igitur sapiens immanissimis cladibus atque succumbit, videlicet tantum ut eis cogatur et in seipsum homicidium perpetrare. Cui iam parcat ut illis careat malis, qui sibi ipse non parcit ? Certe semper beatus est, certe beatam vitam in sua potestate constitutam nulla vi potest ullius calamitatis amittere. Nemo enim, inquit ille, potest non beatissimus esse, qui est totus aptus ex sese, quique in se uno ponit omnia [Cicero, Paradoxa, 2]».

Dans ce passage, Jansénius insiste sur le suicide forcé du sage. En effet, ce dernier cède devant les maux de la vie et ne s'épargne pas soi-même, bien qu'il pense détenir la " clé » d'une vie heureuse. Le théologien de Louvain commente en ces termes :

«O vitam scilicet beatissimam sapientis, qua ut fruatur mortis quærit auxilium! ».

C'est dans le passage ci-dessus que figure une interjection très semblable à celle que Pascal propose dans le fragment L 147. La contradiction du sage stoïcien est ici évidente. Il considère sa vie comme très heureuse, mais préfère demander l'aide de la mort. Jansénius insiste encore sur cette contradiction :

«Si beata est permanet in ea sapiens : si vero propter ista mala fugis ab ea, quomodo est beata? Aut quomodo non sunt mala quæ vincunt tuum illud fortitudinis, hoc est virtutis bonum, quo totus eras aptus ex te et in te uno ponens omnia ? $»^{41}$.

31 Jansénius sème d'abord le doute en suggérant que la vie n'est pas si heureuse qu'on le prétend - sinon, le sage ne la fuirait pas en se tuant. Il met aussi en doute sa force, en contestant qu'elle représente le bien de la vertu (virtutis bonum). En outre, il souligne que les maux de la vie n'entraînent pas seulement la perte de la force du sage, mais aussi qu'ils font de sa sagesse un délire.

32 Comme Jansénius, Pascal, dans le fragment L 147, après la citation de Sénèque, souligne aussi la contradiction des stoïciens à l'égard du suicide : quoiqu'ils exaltent la grandeur de l'homme qui est capable de poursuivre sa quête du bonheur, « ils conseillent enfin de se tuer $^{42} »$. Ceci corrobore l'interjection qui clôt le fragment, par laquelle Pascal commente la thèse stoïcienne :

«Oh! quelle vie heureuse dont on se délivre comme de la peste !»

Or la contradiction des stoïciens relevée par Jansénius et par Pascal à propos du suicide du sage est certainement un thème de l'épître 155 d'Augustin. En effet, celui-ci écrit dans son premier chapitre :

«Ecce in cæcitate et surditate et atrocissimis cruciatibus corporis, aut amisit beatam vitam, aut si et in istis afflictionibus adhuc beata est, fit per huiuscemodi doctissimorum hominum disputationes, ut sit aliquando beata vita, quam sapiens ferre non possit : vel, quod est absurdius, quam sapiens ferre non debeat, eamque fugiat, abrumpat, abiiciat, seseque ab ea ferro etiam seu veneno, vel alia voluntaria morte subducat : ut in portu non sentiendi vel nullus omnino sit, quod Epicurei et si qui alii simili stultitia putaverunt : vel ideo beatus, quoniam fuerit ab illa beata vita, tanquam ab aliqua peste liberatus ${ }^{43} "$.

34 Augustin montre la contradiction des épicuriens et des stoïciens : ils considèrent la vie du sage comme très heureuse; cependant, lorsqu'elle est frappée par des afflictions, ils lui 
conseillent de se tuer pour parvenir "in portu sentiendi ", c'est-à-dire à l'état de tranquillité due à l'absence de sensibilité. Donc, l'analyse de ce passage montre, certes, la présence de la critique des stoïciens (surtout à propos de la contradiction du suicide du sage - motif présent aussi dans le fragment L 147 de Pascal et dans le chapitre VIII du livre II De statu purce naturce de Jansénius).

En conclusion, il me semble que les textes confirment mon assertion de départ : au-delà de la source augustinienne commune à Jansénius et à Pascal, l'élément textuel de l'interjection, présente dans les deux textes examinés, montrerait que Pascal, en composant son fragment, a utilisé, ou tout au moins n'a pas ignoré, le passage de Jansénius.

\section{NOTES}

\section{S $180 ;$ C 375 .}

2. L 140-145 ; B 466, 509, 463, 464, 360, 461 ; C 692, 377, 379, 390, 376, 373 ; S 172-178.

3. L 146 ; B 461; S 179 ; C 374 : « Il concluent qu'on peut toujours ce qu'on peut quelquefois et que, puisque le désir de la gloire fait bien faire à ceux qu'il possède quelque chose, les autres le pourront bien aussi. Ce sont des mouvements fiévreux que la santé ne peut imiter. Épictète conclut de ce qu'il y a des chrétiens constants que chacun le peut bien être [Entr. IV, 7] ».

4. Que les stoïques fassent l'objet de la critique de Pascal et donc des philosophes mis en cause ce que Vincent Carraud a déjà bien montré. Cf. Vincent CARRAud, Pascal et la philosophie, Paris, P.U.F., 1992, ch. II, § 14, p. 201- 213, en part. p. 205.

5. Philippe SELLIER, Pascal et Saint Augustin, Paris, Librairie Armand Colin, 1970.

6. Philippe SELLIER, Pascal et Saint Augustin, cit., p. 83, n. 11 : « Dans le De statu naturce naturce, II, c. 1-9, Jansénius traite de la recherche du souverain bien. C'est à ces forts chapitres que Pascal renvoie par le titre du fragment et celui de la liasse ».

7. Philippe Sellier a signalé la présence de la même citation tirée de Sénèque, aussi bien dans le fragment examiné ici que dans JANSENIUS, Augustinus I, De hæeresi pelagiana V, 1. Cf. Philippe SELLIER, Pascal et Saint Augustin, Paris, Colin, 1970, p. 83, n. 11 et Blaise PASCAL, Pensées, par Philippe Sellier, Paris, Mercure de France, 1976, p. 103, n. 1.

8. Ibidem.

9. Les états du pélagianisme sont les suivants : ethnicisme (HP, l. V, chap. I, cols. 235A-238B ), semi-ethnicisme (HP, l. V, chap. II, cols. 237A-240C), judaïsme (HP, l. V, cap. III, cols. 239A-242C) et semi-christianisme (HP, 1. VI, chap. I, cols. 331A-334C).

10. Le titre de HP V, I est : « Primus Pelagianismi status Ethnicismus: nudam naturam prædicat ». 11. HP V, I, 236B : «Porro primus iste Pelagianismi status nihil est aliud nisi purum putumque Ethnicæ Philosophiæ somnium ut potius Paganismus quam hæresis nuncupari posse videatur ».

12. Ibidem.

13. HP V, I, 236B : « Audi genuinum Philosophum Pelagio præcinentem : Est aliquid quo sapiens antecedat Deum, ille naturce beneficio, non suo sapiens est. Ecce res magna habere imbecillitatem hominis, securitatem Dei [SENECA, Epistola 53] ».

14. HP V , 1, 236B-C. 
15. Par exemple, aussi dans le Liber Procmialis du second tome de l'Augustinus, Jansénius reproche aux pelagiens d'avoir trop donné aux forces de la liberté. LP I, 3A : «Pelagiani sunt, et quotquot eorum dogmatibus vel nunc vel olim implicati fuerunt, qui dum nimium libertatis viribus favent, ad huiusmodi acerba in Christianam fidem sensa aut prudentes delabuntur, aut imprudentes adiguntur » (mon italique).

16. HP V, I, 235B.

17. Philippe SELLIER, Pascal et Saint Augustin, cit., p. 83, n. 11 : « Dans le De statu naturce naturce, II, c. $1-9$, Jansénius traite de la recherche du souverain bien. C'est à ces forts chapitres que Pascal renvoie par le titre du fragment et celui de la liasse ».

18. Voici quelques lieux : SNL IV, X, 597-598 ; SPN IV, XII, 609 ; SPN IV, XIII, 615 ; SPN I, XV, 749 ; SNP II, V, 812 ; SNP III, XV, 951-952.

19. Alberto PERATONER insiste sur le lien entre le souverain bien et le bonheur dans son analyse de la dixième liasse. Cf. Alberto PERATONER, Blaise Pascal: ragione, rivelazione e fondazione dell'etica. Il percorso dell'Apologie, Venezia, Cafoscarina, 2002, en part. pp. 573 ss.

20. L 147.

21. AUGUSTINUS, Epistola LII, dans Opera D. AURELII AUGUSTINI Hipponensis episcopi et doctoris prcecipui, tomis decem comprehensa : per theologos lovanienses ex manuscriptis codicibus multo labore emendata, et $a b$ innumeris erroribus vindicata: illustrata præterea eruditis censuris, et locupletata multis homiliis et aliquot epistolis eiusdem B. Augustini, antea non editis : cum indice tripartito, Antverpiæ, Apud Christophorum Plantinum, 1576-1577 [Lov.], II. Cependant, dans cette édition des théologiens de Louvain, il y a une coquille d'impagination. Aux pages 87-90, on trouve donc le Sermo LXIII In Evangelium Johannis au lieu de l'Epistola LII De vera felicitate, qu'Augustin a écrit en Macédoine dans les années 413-414. J'ai dû consulter la réimpression de 1649 : AUGUSTINUS, Epistola LII, dans Opera tomis undecim comprehensa, per theologos Lovanienses ex vetustissimis codicibus manuscriptis [...] in lucem emissa. Illustrata præterea eruditis censuris, et locupletata multis homiliis et aliquot epistolis eiusdem s. Augustini, antea non editis, Parisiis, Compagnie de la Grande Navire, 1649-1651, II, 89, 1D. Cf. PL 33, 668 ; CSEL 44, 433, 17-18.

22. Le titre de SPN, II, I est le suivant : Secundum argumentum contra statum purce naturce petitur ex fruitione quacunque beatifica summi boni, quce omnes creatas vires superat. Ostenditur primo ex dilectione Dei quam includit.

23. SPN I, III : Primum argumentum contra statum purce naturce ex ordine creaturce rationalis ad Deum ut principium et finem fine cuius amore condi non potest.

24. SPN II, I, 789A.

25. SPN II, I, 789B : «Quod significavit Augustinus cum dixit : In rebus a Deo factis tam magnum bonum est natura rationalis, ut nullum sit bonum quo beata sit nisi Deus [De natura boni 7]».

26. SPN II, I, 789B : «Quod cum in eius natura fundatum sit per quam habet quod est capax Dei, eiusque particeps esse potest, Nec tam magnum bonum nisi per hoc quod imago eius est, intelligi possit [ De Trinitate Dei XIV, 8] ; hinc fit ut, quamdiu eadem ratio imaginis, et capacitas, et consequenter eadem natura permanet, etiam aliter satiata, ac per hoc beata esse non possit nisi Deo ».

27. SPN II, I : Argumentum contra statum purce naturce petitur ex fruitione quacumque beatifica summi boni quce omnes creaturas vires superat. Ostenditur primo ex dilectione Dei quam includit (789A-792B).

28. SPN II, III : Tertium Argumentum quia regnum ccelorum quod supernaturalis ordinis est non potest a pura natura separari sine iniustitia (797A-804C).

29. SPN II, V : Quartum Argumentum ex tribus necessariis ad beatitudinem naturalem non falli, non offendi, non mori ; quce pura natura non potest assequi. De gravissimis erroribus purce naturce (809A-814C).

30. SPN II, X : Quintum et sextum argumentum adversus statum purce naturce, ex ratione liberi arbitrii quo careret ad bonum, et necessitatis peccandi in singulis operibus (825A-828C). 
31. SPN II, VIII : Ostenditur insania Philosophorum naturalem beatitudinem sibi machinantium in ea quam putabant esse puram naturam. Superbia singularis tantum naturce etiam purce viribus tribuere (819A-824B).

32. SPN II, VIII, 819A-820A : « Hæc imaginatio philosophicæ beatitudinis, ex Gentilium librorum consuetudine familiaris facta Christianis et naturali quadam Dei contemplatione condita, quam ei sensus Christianæ pietatis adiecit, naturæ puræ conditores fallere solet ».

33. SPN II, VIII, 819A.

34. SPN II, VIII, 820A.

35. À ce propos, Sellier a signalé que l'expression « sola virtute contentus » a pu rappeler à Pascal le passage de l'Epistola XX de Sénèque, qui est cité par Jansénius dans HP V, I. Cf. Philippe SELLIER, Pascal et Saint Augustin, cit., p. 83, n. 11.

36. SPN II, VIII, 820A-821A.

37. Philippe SEllier, Pascal et Saint Augustin, cit., p. 83, n. 11.

38. Cf. Augustinus, Epistola LII, dans Opera tomis undecim..., cit., 89, 1B : " Unde in errorem absurdissimum lapsi sunt, ut cum asseverant, etiam in Phalaridis tauro beatum esse sapientem, cogantur fateri vitam beatam aliquando esse fugiendam ».

39. SPN II, VIII, 820A-821A.

40. CICERO, Paradoxa stoicorum ad M. Brutum paradoxon, 2, par. 17, p. 202, 1. 5.

41. SPN II, VIII, 821B.

42. L 147. Cf. supra.

43. AUGUSTINUS, Epistola LII, dans Opera tomis undecim comprehensa, cit., II, 89, 1C-D. Cf. PL 33, 668 ; CSEL 44, 433, 8-18.

\section{INDEX}

Mots-clés : Pascal, Jansénius, stoïcisme

Keywords : Pascal, Jansenius, stoicism 